\title{
Clinical significance and comparison of flotillin 1 expression in left and right colon cancer
}

\author{
NADIA BAIG ${ }^{1 *}$, ZEQUN LI $^{2 *}$, JING LU ${ }^{3 *}$, HAIBIN CHEN ${ }^{1}$, SONGYANG YU ${ }^{1}$, \\ TIANEN LI ${ }^{1}$, ZHAOJIAN NIU ${ }^{2}$ and JUN NIU ${ }^{1}$ \\ ${ }^{1}$ Department of General Surgery, Qilu Hospital of Shandong University, Jinan, Shandong 250012; \\ ${ }^{2}$ Department of Gastrointestinal Surgery, Affiliated Hospital of Qingdao University, Qingdao, Shandong 266000; \\ ${ }^{3}$ Department of Chemotherapy, Qilu Hospital of Shandong University, Jinan, Shandong 250012, P.R. China
}

Received November 28, 2017; Accepted September 28, 2018

DOI: $10.3892 / \mathrm{ol} .2019 .10401$

\begin{abstract}
Flotillin 1 (FLOT1) is increasingly implicated in various types of cancer, and has been reported to influence tumorigenesis and cancer progression, leading to poor prognosis for survival time; however, its expression in colorectal cancer (CRC) and its influence on various clinicopathological parameters of this disease remain unknown. In the present study, FLOT1 expression and its effect on different clinicopathological parameters were assessed immunohistochemically and histologically in $81 \mathrm{CRC}$ and 81 non-tumorous colon tissue samples. The immunohistochemical staining was scored semi-quantitatively. The association of FLOT1 expression with various parameters and its effect on overall survival time was also assessed. FLOT1 was upregulated in the CRC tissue, with increased expression in the right colon tissue samples compared with those of left colon. Increased FLOT1 expression in CRC tissue samples was associated with tumor volume, differentiation, tumor grade and poor overall survival time. In the right colon tissue samples in particular, there was a notable association with tumor volume and grade, indicating its effect on proliferation and tumor stage at this site. A multivariate Cox regression hazard analysis revealed that only tumor grade and differentiation were the independent
\end{abstract}

Correspondence to: Dr Zhaojian Niu, Department of Gastrointestinal Surgery, Affiliated Hospital of Qingdao University, 16 Jiangsu Road, Qingdao, Shandong 266000, P.R. China

E-mail: qdfyniuzhaojian@163.com

Dr Jun Niu, Department of General Surgery, Qilu Hospital of Shandong University, 107 Wenhua Xi Road, Jinan, Shandong 250012, P.R. China

E-mail: sduniujun@163.com

*Contributed equally

Abbreviations: CRC, colorectal cancer; ROC, receiver operating characteristic; AUC, area under the curve

Key words: colorectal cancer, right and left colon, flotillin 1, tumor grade, tumor differentiation, tumor volume predictors of overall survival time in patients with CRC. Together, the results of the present study suggest that FLOT1 serves important functions in the proliferation and progression of CRC, contributes to decreased survival time, and may serve as a novel therapeutic target for the treatment of CRC.

\section{Introduction}

Colorectal cancer (CRC) is the third most commonly diagnosed type of cancer and was the second leading cause of cancer-associated mortalities in men and women in 2018 globally. Annually, $>1.2$ million patients are diagnosed with CRC, whereas $>600,000$ mortalities occur due to the disease (1). The development of CRC is primarily associated with the western lifestyle. The prevalence of CRC is higher in males and in individuals aged $>70$ years $(2,3)$. Despite marked hereditary components, the majority of cases of CRC are sporadic and account for $\sim 70 \%$ of all cases. The disease develops slowly over several years through the adenoma-carcinoma sequence. Patients with CRC are primarily diagnosed at an advanced stage of the disease and distant metastases may also be present. Surgically, the procedure for treatment is challenging and the survival rate remains low post-surgery (4). Thus, novel prognostic biomarkers are required to improve the early diagnosis and prognosis of patients with CRC.

That the characteristics and prognoses are different between patients with right-side colon cancer and those with left-side colon cancer remains debatable. Previous studies have identified contrasts in the epidemiology, peri-operative course, pathology and prognosis between patients with cancer of the left side of the colon and those with cancer on the right side (5-7). Patients with right-side colon cancer were typically older and more often female, with tumors of more advanced stages, increased size, poorer differentiation and different molecular patterns, and with a poorer prognosis compared with patients with left-side colon cancer (7). Differences in the embryonic development of the two sides of the colon may partially explain the differences $(8,9)$. In contrast, several studies could not identify any association between tumor location within the colon and the overall survival time (5).

The flotillin (FLOT)/reggie protein family consists of the major constituent proteins of lipid rafts and contains 
two isoforms: FLOT1 and FLOT2 (10). The isoforms form hetero-oligomeric complexes that participate in various cellular functions, including cell adhesion, actin cytoskeleton reorganization, endocytosis, phagocytosis and the transduction of cellular signals (11). FLOT1 is associated with various types of cancer, including non-small cell lung, endometrial and breast cancer, renal cell carcinoma, neuroblastoma, gastric, bladder, mouth, cervical and prostate cancer, and hepatocellular carcinoma (12-22). In breast cancer, the FLOT1 expression level is associated with clinical staging and prognosis, and its silencing inhibits the proliferation and tumorigenicity of breast cancer cells in vitro and in vivo (17). In endometrial cancer, the upregulation of FLOT1 is associated with the tumor grade (12), whereas in bladder cancer it leads to cancer progression and recurrence post-surgery (14). In prostate cancer, increased expression of FLOT1 influences the proliferation of cancer cells (21). These results suggest that FLOT1 may serve an important function in the progression and development of malignant carcinomas. To date, the effect of FLOT1 dysregulation on the pathogenesis of CRC is not well-documented.

Therefore, the aim of the present study was to investigate the expression of FLOT1 in left- and right-side CRC tissue samples, and evaluate its prognostic significance by analyzing the association between the level of FLOT1 expression and clinicopathological features and survival time outcomes from CRC biopsies.

\section{Materials and methods}

Patients and tissue samples. The present study included patients with CRC who were admitted to Qilu Hospital of Shandong University between November 2009 and May 2010. In total, 81 tumor biopsies and adjacent tissues samples were obtained from the patients. The study population consisted of 45 male and 36 female patients with a mean age of $66.7 \pm 14.0$ years (range, 22-93 years). The biopsies were pathologically classified, and the tumor stage was defined according to the World Health Organization (2000) and the Union of International Cancer Control/American Joint Committee on Cancer Tumor-Node-Metastasis (6th edition) classification (23). Following surgical removal, the tumor biopsy samples were formalin-fixed $(10 \%)$ for $24 \mathrm{~h}$ at $4^{\circ} \mathrm{C}$, paraffin-embedded and stored at $4^{\circ} \mathrm{C}$ for further histological analysis and immunohistological studies. The post-surgery clinical follow-up of the patients took place in the surgical outpatient clinic biannually for $\leq 68$ months. The age, sex, tumor location, tumor size, histological type, tumor-node-metastasis stage, and presence of lymph node and distant metastases were obtained from the medical records of the patients. The Ethics Committee of Shandong University, China approved the study. Written informed consent was obtained from each patient.

Immunohistochemistry. The paraffin-embedded tissue sections were cut into 4-5- $\mu \mathrm{m}$ sections, dewaxed and hydrated. The antigen retrieval was performed by heating the tissue sections at $95^{\circ} \mathrm{C}$ in $0.01 \mathrm{M}$ citrate buffer solution, $\mathrm{pH}$ 6.0, for 2-3 min in a stainless steel pressure cooker. The endogenous peroxidase was blocked for $10 \mathrm{~min}$ using $3 \%$ hydrogen peroxide solution at room temperature. The sections were incubated with goat serum (Beijing Solarbio Science \& Technology Co., Ltd., Beijing, China) for $15 \mathrm{~min}$ at $37^{\circ} \mathrm{C}$ for blocking and then immunostained with rabbit antibody against FLOT1 (cat. no. ab41927; dilution 1:100; Abcam) at $4^{\circ} \mathrm{C}$ overnight. Incubation with horseradish peroxidase-conjugated anti-rabbit immunoglobulin G (cat. no. A0208; dilution 1:50; Beyotime Institute of Biotechnology, Haimen, China) for $1 \mathrm{~h}$ at $37^{\circ} \mathrm{C}$, followed by incubation with 3,3'-diaminobenzidine (DAB-0031; Fuzhou Maixin Biotech Co., Ltd., Fuzhou, China) for $1 \mathrm{~min}$ at room temperature for secondary staining, according to the manufacturer's protocols. Hematoxylin was used for counterstaining for $1 \mathrm{~min}$ at room temperature.

Evaluation of immunohistochemical staining. Immunostaining was assessed independently by two experienced pathologists in a blinded manner. The concurrence ratio was $>95 \%$ and when discrepancies appeared, a third pathologist analyzed the results. Overall, 500 cells from three randomly chosen fields under the light microscope (magnification, x200) were counted for each sample. The scoring for immunostaining was performed semi-quantitatively: A combined score of the staining intensity ( 0 , negative; 1 , weak; 2 , moderate; and 3 , strong) and the percentage of positively stained cells ( 0,0 ; $1,1-25 ; 2,26-50 ; 3,51-75$; and 4, 76-100\%) was calculated, giving the final score of FLOT1 protein expression. The cut-off point for the definition of the expression level was as follows: 0-5, low expression; and 6-8, high expression.

Statistical analysis. The associations between FLOT-1 expression and clinicopathological parameters were analyzed using $\chi^{2}$ and Fisher's exact tests. Quantitative data are presented as the mean \pm standard deviation. Paired and unpaired Student's t-tests were used to determine the differences between two groups to compare FLOT-1 expression in adjacent normal tissues and colon cancer tissues or left and right colon cancer tissues). Receiver operating characteristic (ROC) curve analysis was performed to assess the diagnostic value of FLOT1 in $\mathrm{CRC}$. The survival rates were calculated by the Kaplan-Meier method and the differences between the subgroups were determined using the log-rank test. Univariate and multivariate survival analyses were performed using Cox proportional hazards models to identify independent prognostic factors. All statistical analyses were performed using SPSS software (version 21.0; IBM Corp., Armonk, NY, USA), and P<0.05 was considered to indicate a statistically significant difference.

\section{Results}

Expression of FLOT1 in CRC. FLOT1 staining was markedly increased in the CRC tissues compared with their corresponding adjacent non-cancer tissues, suggesting that FLOT1 is upregulated in CRC ( $\mathrm{P}<0.001$; Figs. 1 and $2 \mathrm{~A})$. Of the 81 CRC tissues, 45 (56\%) demonstrated strong positive staining, whereas $36(44 \%)$ revealed either negative or weak staining. In the adjacent healthy tissues, 81 (100\%) samples demonstrated either negative or weak staining and none revealed strong positive staining. Among the CRC samples, the FLOT1 levels were significantly higher in samples from the right side of the colon compared with those from the left side, indicating a differential expression of FLOT1 in CRC location ( $\mathrm{P}<0.05$; Fig. 2B). 
A

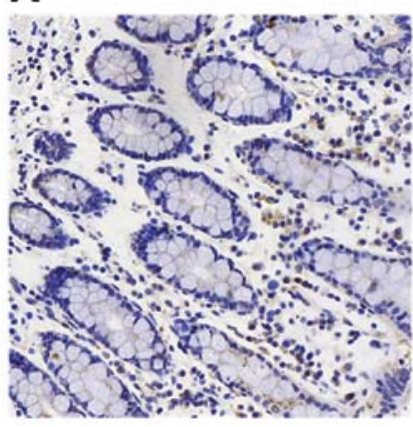

B

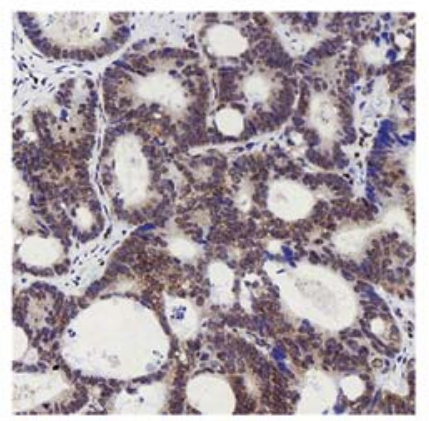

C

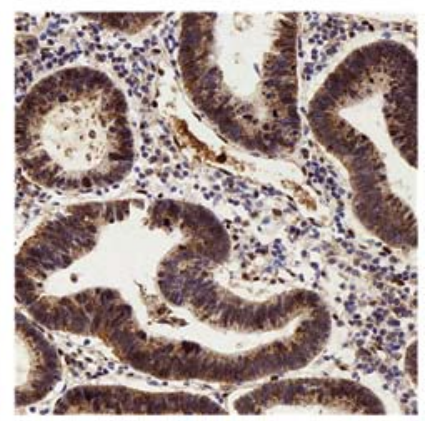

Figure 1. FLOT1 expression in healthy colon tissue and cancer tissues of the left and right colon. (A) Tumor-adjacent normal tissue with negative staining for FLOT1. (B) Moderate staining for FLOT1 was observed in mucosal layer of the tumor in the left colon. (C) Strong positive staining for FLOT1 was observed in the mucosal layer of tumor in the right colon. Original magnification, x200. FLOT1, flotillin 1.

A

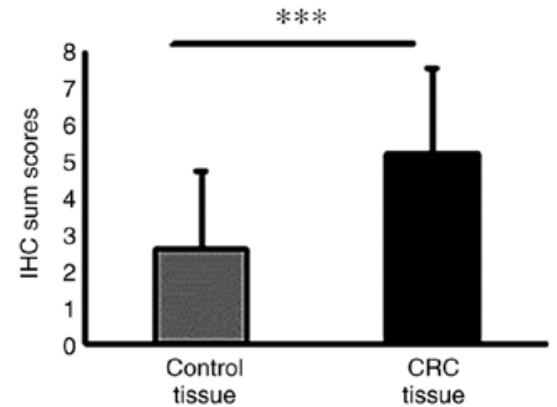

C

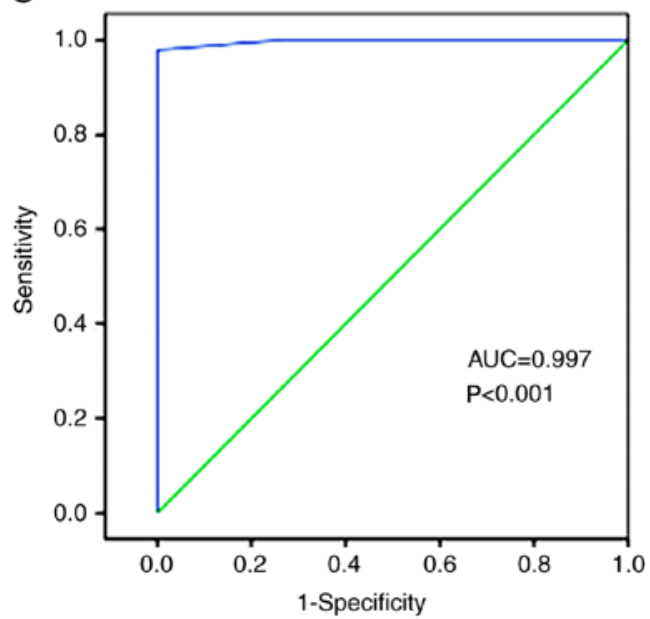

B

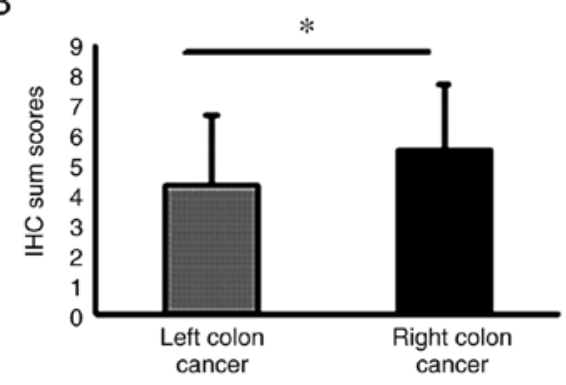

D

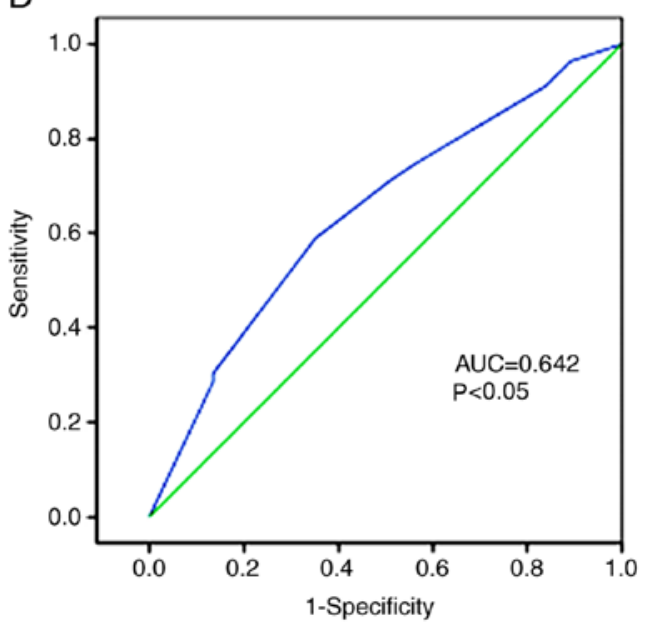

Figure 2. Expression of FLOT1 in CRC biopsy samples. (A) FLOT1 expression was significantly upregulated in CRC tissues compared with the adjacent healthy tissues. (B) FLOT1 expression was significantly higher in the right colon cancer tissues compared with those of the left colon. The receiver operating characteristic curves indicate a marked difference (C) between $C R C$ tissues and adjacent non-cancer controls (AUC $=0.997$; $P<0.001$ ), (D) and between right and left colon cancer tissues ( $\mathrm{AUC}=0.642 ; \mathrm{P}<0.05$ ). The green diagonal line is the reference line and the blue line indicates the threshold values. ${ }^{* * * *} \mathrm{P}<0.001$ and ${ }^{*} \mathrm{P}<0.05$. FLOT1, flotillin 1; CRC, colorectal cancer; IHC, immunohistochemistry; AUC, area under the curve.

To determine the diagnostic value of FLOT1 expression in CRC and in different CRC sites, ROC curves were constructed. To assess the potential of the FLOT1 expression (IHC sum scores) for diagnosing CRC and its location, the area under the curve (AUC) was calculated. The AUC of the ROC curve for FLOT1 as a predictor of CRC reached 0.997 [95\% confidence interval (CI), 0.99-1.00; P<0.001; Fig. 2C], with an estimated sensitivity and specificity of 100 and $87.0 \%$, respectively. Furthermore, the AUC for FLOT1 being able to predict the CRC location was 0.642 (95\% CI, 0.53-0.76; P<0.05; Fig. 2D) with an estimated sensitivity and specificity of 96.4 and $89.2 \%$, respectively. However, the FLOT1 expression was comparable between the tumor tissues with and without lymph node metastasis ( $\mathrm{P}=0.473$; Fig. 3).

Association between FLOT1 expression and clinicopathological factors. To estimate the clinical importance of FLOT1 expression in CRC, the association between FLOT1 levels and various clinicopathological factors was analyzed (Table I). FLOT1 expression was significantly associated with tumor invasiveness $(\mathrm{P}=0.025)$, grade $(\mathrm{P}=0.047)$ and differentiation $(\mathrm{P}=0.023)$, and the association with tumor location was 
Table I. Association between FLOT1 expression and clinicopathological factors in colorectal cancer.

\begin{tabular}{|c|c|c|c|c|}
\hline \multirow[b]{2}{*}{ Variable } & \multirow[b]{2}{*}{ Cases, $\mathrm{n}$} & \multicolumn{2}{|c|}{ FLOT1 expression } & \multirow[b]{2}{*}{ P-value } \\
\hline & & Low & High & \\
\hline Age, years & & & & 0.335 \\
\hline$<60$ & 25 & 9 & 16 & \\
\hline$\geq 60$ & 56 & 28 & 28 & \\
\hline Sex & & & & 1.000 \\
\hline Male & 45 & 21 & 24 & \\
\hline Female & 36 & 16 & 20 & \\
\hline Depth of invasion (T) & & & & 0.025 \\
\hline $\mathrm{T} 2$ & 5 & 4 & 1 & \\
\hline $\mathrm{T} 3$ & 56 & 25 & 31 & \\
\hline $\mathrm{T} 4$ & 12 & 2 & 10 & \\
\hline Missing data & 8 & 6 & 2 & \\
\hline Lymph node metastasis $(\mathrm{N})$ & & & & 0.149 \\
\hline Negative (N0) & 45 & 24 & 21 & \\
\hline Positive (N1-N2) & 33 & 13 & 20 & \\
\hline Missing data & 3 & 0 & 3 & \\
\hline Distant metastasis (M) & & & & 0.246 \\
\hline Negative (M0) & 78 & 37 & 41 & \\
\hline Positive (M1) & 3 & 0 & 3 & \\
\hline Tumor stage & & & & 0.047 \\
\hline I & 4 & 0 & 4 & \\
\hline II & 36 & 16 & 20 & \\
\hline III & 29 & 11 & 18 & \\
\hline IV & 3 & 0 & 3 & \\
\hline Missing data & 9 & 6 & 3 & \\
\hline Tumor differentiation & & & & 0.023 \\
\hline Well & 1 & 1 & 0 & \\
\hline Moderate & 67 & 34 & 33 & \\
\hline Poor & 13 & 2 & 11 & \\
\hline Tumor location & & & & 0.021 \\
\hline Right & 51 & 18 & 33 & \\
\hline Left & 30 & 19 & 11 & \\
\hline
\end{tabular}

FLOT1, flotillin 1 .

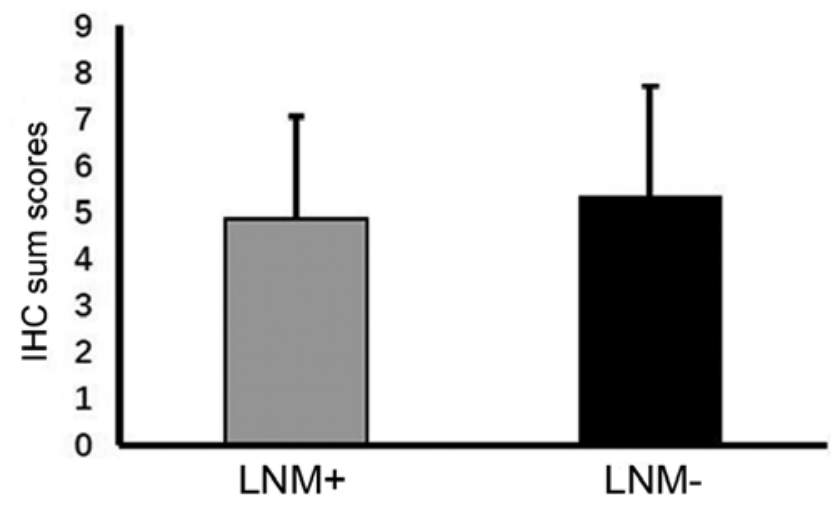

Figure 3. Expression of flotillin 1 in colorectal cancer tissues with and without LNM. IHC, immunohistochemistry; LNM, lymph node metastasis. confirmed $(\mathrm{P}=0.021)$. However, no association was observed between FLOT1 expression and patient age $(\mathrm{P}=0.335)$, sex $(\mathrm{P}=1.00)$, lymph node metastasis $(\mathrm{P}=0.446)$ or distant metastasis $(\mathrm{P}=0.246)$. Furthermore, the association between the FLOT1 levels and clinicopathological factors of the left- compared with the right-side CRC tissue samples was examined (Table II). The analysis revealed an association between the FLOT1 expression and tumor differentiation $(\mathrm{P}=0.025)$ only. No tumor location-dependent association was observed between the other parameters and FLOT1 expression (Table II). Furthermore, although the tumor volume was comparable between CRC tissue samples with low and high FLOT1 levels, it was markedly increased in right- compared with left-side colon cancer tissues (Figs. 4 and 5). These data imply that 
Table II. Association between FLOT1 proteins levels and clinicopathological factors in right- compared with left-side colon cancer tissue samples.

\begin{tabular}{|c|c|c|c|c|}
\hline \multirow[b]{2}{*}{ Variable } & \multirow[b]{2}{*}{ Cases, $\mathrm{n}$} & \multicolumn{2}{|c|}{ FLOT1 expression } & \multirow[b]{2}{*}{ P-value } \\
\hline & & Left & Right & \\
\hline Age, years & & & & 0.805 \\
\hline$<60$ & 25 & 10 & 15 & \\
\hline$\geq 60$ & 56 & 20 & 36 & \\
\hline Sex & & & & 0.645 \\
\hline Male & 45 & 18 & 27 & \\
\hline Female & 36 & 12 & 24 & \\
\hline Depth of invasion $(\mathrm{T})$ & & & & 1.000 \\
\hline $\mathrm{T} 2$ & 5 & 2 & 3 & \\
\hline $\mathrm{T} 3$ & 56 & 21 & 35 & \\
\hline $\mathrm{T} 4$ & 12 & 4 & 8 & \\
\hline Missing data & 8 & 3 & 5 & \\
\hline Lymph node metastasis $(\mathrm{N})$ & & & & 0.326 \\
\hline Negative (N0) & 45 & 19 & 26 & \\
\hline Positive (N1-N2) & 33 & 11 & 22 & \\
\hline Missing data & 3 & 0 & 3 & \\
\hline Distant metastasis $(\mathrm{M})$ & & & & 1.000 \\
\hline Negative (M0) & 78 & 29 & 49 & \\
\hline Positive (M1) & 3 & 1 & 2 & \\
\hline Tumor stage & & & & 0.664 \\
\hline I & 4 & 2 & 2 & \\
\hline II & 36 & 16 & 20 & \\
\hline III & 29 & 9 & 20 & \\
\hline IV & 3 & 1 & 2 & \\
\hline Missing data & 9 & 2 & 7 & \\
\hline Tumor differentiation & & & & 0.025 \\
\hline Well & 1 & 1 & 0 & \\
\hline Moderate & 67 & 29 & 38 & \\
\hline Poor & 13 & 1 & 12 & \\
\hline
\end{tabular}

FLOT1, flotillin 1.

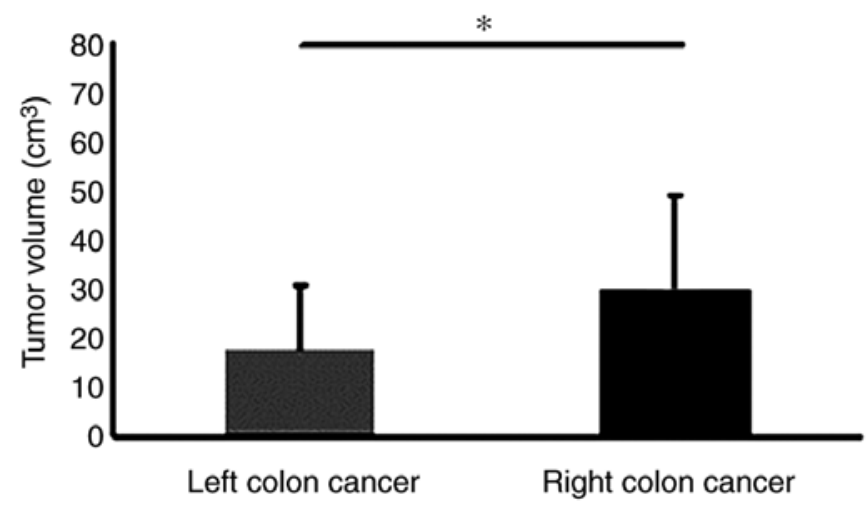

Figure 4. Comparison of tumor volume between colon cancer tumors located on the left and right side of the colon. The tumor volume was higher in colon cancer of the right side compared with tumors on the left side of the colon. ${ }^{*} \mathrm{P}<0.05$.

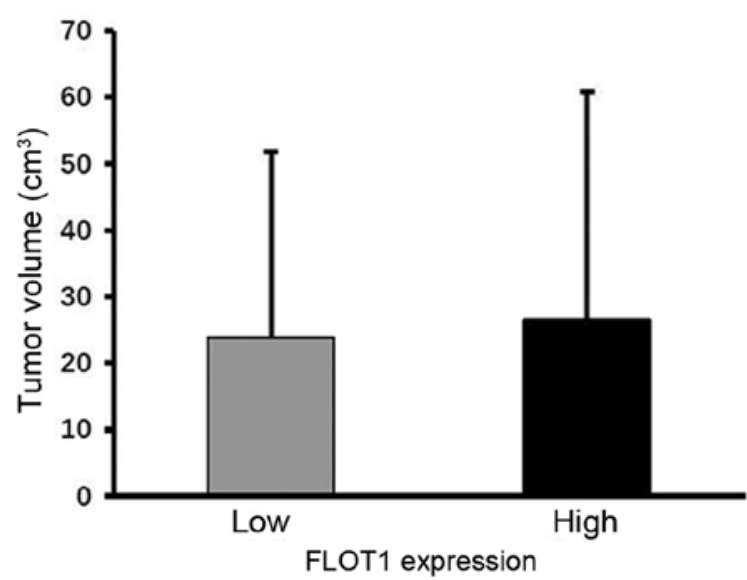

Figure 5. Comparison of the tumor volume of CRC tissues with high and low expression of the flotillin 1 protein. 

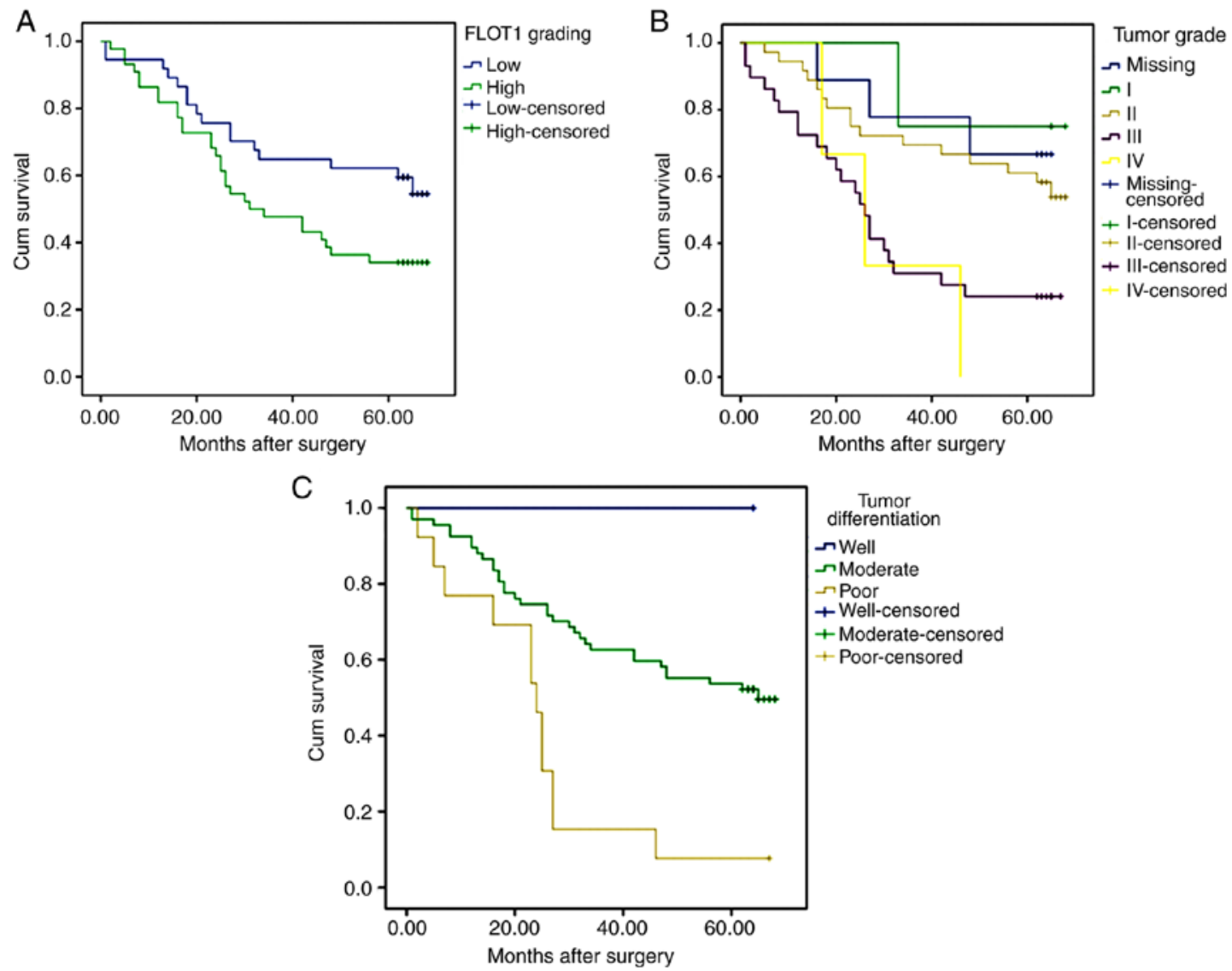

Figure 6. Kaplan-Meier survival curves of patients with colorectal cancer. A log-rank test revealed the significant effect of (A) tumor FLOT1 protein levels $(\mathrm{P}=0.043)$, (B) tumor grade $(\mathrm{P}=0.004)$ and $(\mathrm{C})$ the level of differentiation of the tumor $(\mathrm{P}=0.001)$ on the overall survival time of the patients. FLOT1, flotillin 1; Cum, cumulative.

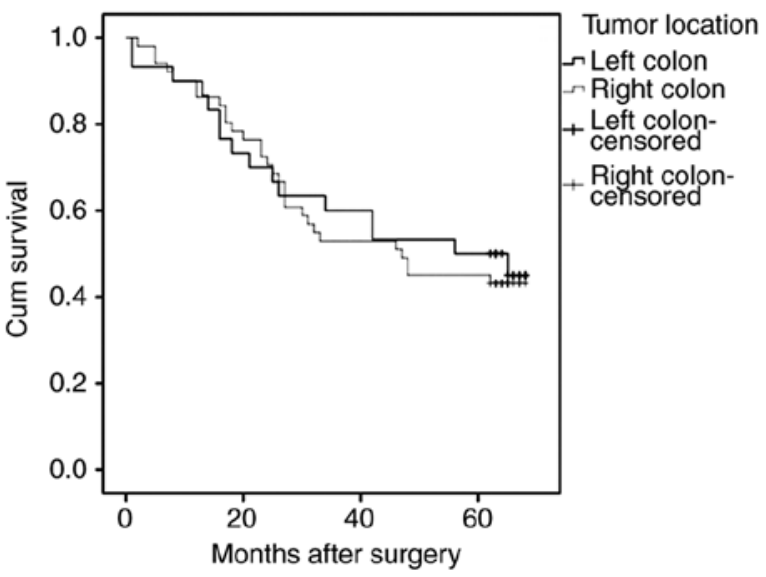

Figure 7. Kaplan-Meier survival curves illustrating no significant effect of the tumor location (right and left side of the colon) on the overall survival times of patients with colorectal cancer. Cum, cumulative.

increased FLOT1 expression leads to tumor progression in CRC in general, whereas it specifically increases proliferation in CRC of the right side of the colon.

FLOT1 as a marker for prognosis. The overall median survival time of the patients in the present study was 48 months (range, 1-68 months). The prognostic value of high FLOT1 expression in samples of patients with CRC was assessed using
Kaplan-Meier analysis. The survival time analysis revealed that patients with low FLOT1 expression in their biopsy samples exhibited longer overall survival times compared with those with high FLOT levels $(\mathrm{P}=0.043$; Fig. 6A). Furthermore, patients with higher tumor grade and poorer tumor differentiation exhibited decreased overall survival times compared with those with lower tumor grade $(\mathrm{P}=0.004$; Fig. 6B) and better-differentiated tumors ( $\mathrm{P}=0.001$; Fig. 6C), respectively. No significant association was revealed between the tumor location (right or left side of the colon) and the overall survival time (Fig. 7). However, the survival estimates demonstrated a lower median survival duration of 44 months for patients with CRC tumors on the right side of the colon (95\% CI, 16.01-77.98) compared with a median survival time of 56 months for patients with left-side tumors $(95 \% \mathrm{CI}$, 19.02-92.97).

Univariate and multivariate analyses using the Cox proportional hazards regression model were performed to further investigate factors associated with patient outcome. The univariate analysis indicated that tumor stage, differentiation and volume were significantly associated with the overall survival time of patients with CRC (Table III). However, FLOT1 expression, tumor location, lymph node metastasis, distant metastasis and depth of invasion indicated no prognostic effect in the patients. The multivariate analysis confirmed tumor stage and differentiation as independent prognostic factors for CRC survival time (Table III). 
Table III. Univariate and multivariate Cox proportional hazard analyses of survival times of patients with colorectal cancer.

\begin{tabular}{|c|c|c|c|c|c|c|}
\hline \multirow[b]{2}{*}{ Variable } & \multicolumn{3}{|c|}{ Univariate analysis } & \multicolumn{3}{|c|}{ Multivariate analysis } \\
\hline & HR & $95 \% \mathrm{CI}$ & P-value & HR & $95 \% \mathrm{CI}$ & P-value \\
\hline FLOT1 expression & 1.084 & $0.588-1.996$ & 0.797 & 1.333 & $0.666-2.669$ & 0.416 \\
\hline Depth of invasion & 1.383 & $0.947-2.020$ & 0.093 & 1.070 & $0.696-1.646$ & 0.757 \\
\hline Lymph node metastasis & 1.518 & $0.977-2.359$ & 0.063 & 1.320 & $0.692-2.333$ & 0.400 \\
\hline Distant metastasis & 2.430 & $0.746-7.914$ & 0.141 & 0.410 & $0.095-1.768$ & 0.232 \\
\hline Tumor stage & 1.799 & $1.259-2.572$ & 0.001 & 1.587 & $1.004-2.508$ & 0.048 \\
\hline Tumor differentiation & 3.438 & $1.760-6.716$ & 0.000 & 2.520 & $1.042-6.093$ & 0.040 \\
\hline Tumor volume & 1.008 & $1.001-1.015$ & 0.032 & 1.004 & $0.995-1.014$ & 0.345 \\
\hline Tumor location & 1.084 & $0.588-1.996$ & 0.797 & 0.724 & $0.36-1.452$ & 0.363 \\
\hline
\end{tabular}

FLOT1, flotillin 1; HR, hazard ratio; CI, confidence interval.

\section{Discussion}

The lipid raft marker protein FLOT1 is upregulated in various types of cancer.Previously, Thorn et al (24) performed expression profiling in the marginal edges of CRC tumors and demonstrated that FLOT1 expression is increased in the more invasive cancer tissues. In accordance with this study, the results of the present study demonstrated that FLOT1 expression was significantly increased in CRC tissues, with increased expression specifically in the right-side tumors, and was associated with tumor differentiation, tumor grade and depth of tumor invasion. Another landmark study by Niu et al (25) suggested the active involvement of FLOT1 and histone H1 as downstream factors in the cytoplasmic and nuclear pathway of the S100 calcium-binding protein A11 (S100A11), and that they are required for LIM and SH3 protein 1 (LASP1)-S100A11 axis-mediated epithelial-mesenchymal transition and $\mathrm{CRC}$ progression. This previous study suggested that S100A11, in combination with LASP1, serves an important function in CRC metastasis through its subcellular effectors FLOT1 and histone $\mathrm{H} 1$.

In various types of cancer, FLOT1 expression is associated with different clinicopathological parameters, including tumor size and tumor stage. Pust et al (26) identified that FLOT1 activates ErbB2 receptor tyrosine kinase 2 expression in vitro and in vivo, which in turn increases the proliferation of breast cancer cells, whereas decreasing FLOT1 expression significantly suppresses breast cancer cell proliferation. In another study, Zhang et al demonstrated an association between FLOT1 and tumorigenesis and progression of hepatocellular carcinoma. In addition, the study also suggested that FLOT1 may be used as a prognostic marker in patients with hepatocellular carcinoma (20). Winship et al revealed upregulation of FLOT1 in endometrial cancer tissue and its association with increasing tumor grade (12). In tongue squamous cell cancer, the FLOT1 protein is correlated with pathological stage, depth of invasiveness, lymph node metastasis, recurrence following the surgical removal of the cancerous tissue, and with shorter survival time (15). In the present study, the association of increased FLOT1 expression with higher tumor grade, poorer differentiation state and increased tumor volume indicates that FLOT1 expression may contribute to the progression of the tumor from early to advanced phenotype, and to the proliferation of CRC cells. Decreased overall survival time was also associated with increased FLOT1 expression. Multivariate analysis confirmed tumor grade and differentiation as independent predictors of overall survival time in patients with CRC.

The notion that patients with tumors located on the right side of the colon have more advanced tumors with poorer prognosis and decreased overall survival time, remains unclear. Although a number of studies have supported this hypothesis $(27,28)$, others failed to demonstrate any site-specific differences $(5,29)$. In the present study, FLOT1 protein levels were significantly increased in right-side colon CRC tissue samples compared with those from the left side. In concordance, the tumor volume was also higher in the right-side colon CRC tissue samples. No other examined parameters differed between the CRC tumors from different locations. This suggests that FLOT1 may affect the proliferation of cancer cells on the right side of the colon. However, in the multivariate analysis, the tumor location failed to predict the overall survival time outcome. This could be due to the small sample size in the present study. Although this is a notable result, further study of this phenomenon is required.

In conclusion, the FLOT1 protein level is increased in CRC tissue compared with in adjacent healthy colon tissue, with tissue from tumors of the right side of the colon specifically exhibiting higher FLOT1 expression. FLOT1 in CRC tissues is associated with the tumor proliferation, differentiation, tumor grade and overall survival time. In right-side colon tumors, FLOT1 specifically affects tumor proliferation. Since, to the best of our knowledge, the present study is the first of its type, the factors investigated should be taken into consideration by the physician when screening patients for CRC. Further studies with larger study sample numbers are warranted, to elucidate the pathological molecular mechanism of this event.

\section{Acknowledgements}

Not applicable. 


\section{Funding}

The present study was supported by the National Natural Science Foundation (grant no. 81572414).

\section{Availability of data and materials}

The datasets used and analyzed for the present study are available from the corresponding author upon reasonable request.

\section{Authors' contributions}

JN and ZL designed the study. NB and JL performed the experiments and wrote the initial draft of the manuscript. HC, SY and TL contributed to the analysis and interpretation of data. $\mathrm{ZN}$ revised the paper, assisted with the experiments and supervised the experimental work. All authors approved the final manuscript.

\section{Ethics approval and consent to participate}

The present study has been approved by the Ethics Committee of Shandong University, Jinan, China (KYLL-2005(KS)-114). All the research works have been conducted in accordance with the Declaration of Helsinki.

\section{Patient consent for publication}

Not applicable.

\section{Competing interests}

The authors declare that they have no competing interests.

\section{References}

1. Bray F, Ferlay J, Soerjomataram I, Siegel RL, Torre LA and Jemal A: Global cancer statistics 2018: GLOBOCAN estimates of incidence and mortality worldwide for 36 cancers in 185 countries. CA Cancer J Clin 68: 394-424, 2018.

2. Siegel RL, Miller KD and Jemal A: Cancer statistics, 2018. CA Cancer J Clini 68: 7-30, 2018.

3. Tuan J and Chen YX: Dietary and lifestyle factors associated with colorectal cancer risk and interactions with microbiota: Fiber, red or processed meat and alcoholic drinks. Gastrointest Tumors 3: 17-24, 2016.

4. Laohavinij S, Maneechavakajorn J and Techatanol P: Prognostic factors for survival in colorectal cancer patients. J Med Assoc Thai 93: 1156-1166, 2010.

5. Hansen IO and Jess P: Possible better long-term survival in left versus right-sided colon cancer - A systematic review. Dan Med J 59: A4444, 2012.

6. Wray CM, Ziogas A, Hinojosa MW, Le H, Stamos MJ and Zell JA Tumor subsite location within the colon is prognostic for survival after colon cancer diagnosis. Dis Colon Rectum 52: 1359-1366, 2009

7. Benedix F, Kube R, Meyer F, Schmid U, Gastinger I and Lippert H; Colon/Rectum Carcinomas and (Primary Tumor) Study Group: Comparison of 17,641 patients with right- and left-sided colon cancer: Differences in epidemiology, perioperative course, histology, and survival. Dis Colon Rectum 53: 57-64, 2010.

8. Hutchins G, Southward K, Handley K, Magill L, Beaumont C, Stahlschmidt J, Richman S, Chambers P, Seymour M, Kerr D, et al: Value of mismatch repair, KRAS, and BRAF mutations in predicting recurrence and benefits from chemotherapy in colorectal cancer. J Clini Oncol 29: 1261-1270, 2011.

9. Elnatan J, Goh HS and Smith DR: C-KI-RAS activation and the biological behaviour of proximal and distal colonic adenocarcinomas. Eur J Cancer 32A: 491-497, 1996.
10. Babuke T and Tikkanen R: Dissecting the molecular function of reggie/flotillin proteins. Eur J Cell Biol 86: 525-532, 2007.

11. Langhorst MF, Reuter A and Stuermer CA: Scaffolding microdomains and beyond: The function of reggie/flotillin proteins. Cell Mol Life Sci 62: 2228-2240, 2005.

12. Winship AL, Rainczuk K and Dimitriadis E: Flotillin-1 protein is upregulated in human endometrial cancer and localization shifts from epithelial to stromal with increasing tumor grade. Cancer Investigat 34: 26-31, 2016.

13. Yang FQ, Zhang HM, Chen SJ, Yan Y and Zheng JH: MiR-506 is down-regulated in clear cell renal cell carcinoma and inhibits cell growth and metastasis via targeting FLOT1. PLoS One 10: e0120258, 2015.

14. Guan Y, Song H, Zhang G and Ai X: Overexpression of flotillin-1 is involved in proliferation and recurrence of bladder transitional cell carcinoma. Oncol Rep 32: 748-754, 2014.

15. Li H, Zhang Y, Chen SW, Li FJ, Zhuang SM, Wang LP, Zhang J and Song M: Prognostic significance of Flotillin1 expression in clinically N0 tongue squamous cell cancer. Int J Clini Exp Pathol 7: 996-1003, 2014.

16. Gao W, Xu J, Wang F, Zhang L, Peng R, Shu Y, Wu J, Tang Q and Zhu Y: Plasma membrane proteomic analysis of human gastric cancer tissues: Revealing flotillin 1 as a marker for gastric cancer. BMC Cancer 15: 367, 2015.

17. Li L, Luo J, Wang B, Wang D, Xie X, Yuan L, Guo J, Xi S, Gao J, Lin X, et al: Microrna-124 targets flotillin-1 to regulate proliferation and migration in breast cancer. Mol Cancer 12: 163, 2013.

18. Arkhipova KA, Sheyderman AN, Laktionov KK, Mochalnikova VV and Zborovskaya IB: Simultaneous expression of flotillin-1, flotillin-2, stomatin and caveolin-1 in non-small cell lung cancer and soft tissue sarcomas. BMC Cancer 14: 100, 2014.

19. Li Z, Yang Y, Gao Y, Wu X, Yang X, Zhu Y, Yang H, Wu L, Yang C and Song L: Elevated expression of flotillin-1 is associated with lymph node metastasis and poor prognosis in early-stage cervical cancer. Ameri J Cancer Res 6: 38-50, 2015.

20. Zhang SH, Wang CJ, Shi L, Li XH, Zhou J, Song LB and Liao WT: High expression of FLOT1 is associated with progression and poor prognosis in hepatocellular carcinoma. PLoS One 8: e64709, 2013.

21. Gomez V, Sese M, Santamaria A, Martínez JD, Castellanos E, Soler M, Thomson TM and Paciucci R: Regulation of aurora B kinase by the lipid raft protein flotillin-1. J Biological Chem 285: 20683-20690, 2010.

22. Ou YX, Liu FT, Chen FY and Zhu ZM: Prognostic value of Flotillin-1 expression in patients with solid tumors. Oncotarget 8: 52665-52677, 2017.

23. Kim SH, Ha TK and Kwon SJ: Evaluation of the 7th AJCC TNM staging system in point of lymph node classification. J Gastric Cancer 11: 94-100, 2011.

24. Thorn CC, Freeman TC, Scott N, Guillou PJ and Jayne DG: Laser microdissection expression profiling of marginal edges of colorectal tumours reveals evidence of increased lactate metabolism in the aggressive phenotype. Gut 58: 404-412, 2009.

25. Niu Y, Shao Z, Wang H, Yang J, Zhang F, Luo Y, Xu L, Ding Y and Zhao L: LASP1-S100A11 axis promotes colorectal cancer aggressiveness by modulating TGF $\beta /$ Smad signaling. Sci Rep 6: 26112, 2016.

26. Pust S, Klokk TI, Musa N, Jenstad M, Risberg B, Erikstein B, Tcatchoff L, Liest $\varnothing 1 \mathrm{~K}$, Danielsen HE, van Deurs B, et al: Flotillins as regulators of ErbB2 levels in breast cancer. Oncogene 32: 3443-3451, 2013.

27. Nawa T, Kato J, Kawamoto H, Okada H, Yamamoto H, Kohno H, Endo H and Shiratori Y: Differences between right- and left-sided colon cancer in patient characteristics, cancer morphology and histology. J Gastroenterol Hepatol 23: 418-423, 2008.

28. Rothberg PG, Spandorfer JM, Erisman MD, Staroscik RN, Sears HF, Petersen RO and Astrin SM: Evidence that c-myc expression defines two genetically distinct forms of colorectal adenocarcinoma. Br J Cancer 52: 629-632, 1985.

29. Gervaz P, Bouzourene H, Cerottini JP, Chaubert P, Benhattar J, Secic M, Wexner S, Givel JC and Belin B: Dukes B colorectal cancer: Distinct genetic categories and clinical outcome based on proximal or distal tumor location. Dis Colon Rectum 44: 364-372, 2001.

This work is licensed under a Creative Commons Attribution-NonCommercial-NoDerivatives 4.0 International (CC BY-NC-ND 4.0) License. 